\title{
Visualizing and Comparing Online Travel Reviews of the Great Walls: A Data Mining Approach
}

\author{
Jin Ling and Nadezda Sorokina ${ }^{(凶)}(\mathbb{D}$ \\ SIHOM, Woosong University, Dongdaejon-ro 171, \\ Dong-gu, Daejeon 34606, South Korea \\ Nadia_sorokina@sis.ac.kr
}

\begin{abstract}
This research employs two samples of heritage sites of the Great Wall of China (Ba daling Great Wall and Mu tianyu Great Wall) and their 21000 reviews on TripAdvisor to visualize and induce feature-related comparisons. Word2vec and D3.js are applied for statistical computing and graphing Minimal Spanning Tree (MST) and ThemeRiver. The applications of MST and ThemeRiver are used to delineate outstanding features and clearer feature relationships. In terms of methodology, we applied an innovative research route to combine MST with ThemeRiver to visualize travellers' online comments. At the same time, the visual results obtained are combined with qualitative analysis to generate valuable, intuitive summaries that can be used for reference in future research. Practically, the results disclose that although both sites are highly enjoyed by tourists, they are significantly different in terms of service, infrastructure and scenery. This article has implications for policymakers and practitioners with regard to making use of online reviews to gather authentic visitor comments on the Great Wall.
\end{abstract}

Keywords: Travel reviews $\cdot$ Minimal Spanning Tree $\cdot$ ThemeRiver $\cdot$ The Great Wall of China

\section{Introduction}

The Great Wall of China is an ancient series of walls and fortifications, totaling more than 13,000 miles in length, located in northern China with a history of over 2000 years [1]. Today, the Great Wall is generally recognized as one of the most impressive architectural feats in human history, and UNESCO (1987) designated is as a World Heritage site. Mu tianyu Great Wall and Ba daling Great Wall in Beijing, China, are two heritage sites that attract millions of national and foreign tourists every year.

Given that there is a limited number of prior studies in the existing tourism literature on the comparisons of similar heritage sites through a data mining approach, and only a few scholars have been working to achieve feature visualization of travel reviews in the business application [2-7] . By delineating the Great Wall's outstanding features and clearer feature-related term relationships and showing the differences between the two heritage sites in reviews, this study provides practical suggestions for heritage site operators to improve (instead of better) their performances.

(C) The Author(s) 2022 


\subsection{Research Questions}

This research focuses on a description of the review corpus: (1) identifying what feature-related terms tourists concern most in travel reviews, and (2) exploring terms through Minimal Spanning Tree (MST) and focusing on how the most used adjective terms flow in ThemeRiver and extracting implications from it. (3) assessing if the findings can be used to enhance attraction services.

\subsection{Literature Review and the Premise}

"Words mentioned most frequently carry the main source of elements that determine either a positive or a negative experience" [8]. From top 50 terms from Mu tianyu Great Wall [9] and Ba daling Great Wall [10] reviews, the distribution of notional verbs, nouns, adjectives, and adverbs is highly skewed in that there are a relatively small number of terms with high frequencies. While counting the term frequency is useful in reviews extraction, which gives an outlook of tourists' concerns, examining the distribution of the terms in the documents concerning other terms can be more powerful to understand the contexts in which the terms are used. Structures are here based on the premise that: (1) people organize their beliefs depending on travel experiences into patterns that reflect term relationships, and (2) the most important things are written first.

\section{Data Preprocessing and Data Process Flow}

This article mainly uses the Natural Language Toolkit for language data classification (which divides a large piece of text to units), tokenization, stemming (which shares words with inflection in them to root forms), tagging, parsing (grammatical analysis for a sentence), and semantic reasoning [11].

The collected review texts are first saved into an excel document. Word2vec and D3.js are applied for statistical computing and graphing MST and ThemeRiver. Word2vec makes natural language computer-readable [12]. And each word of this dictionary is fed into D3.js to draw MST and ThemeRiver (Fig. 1).

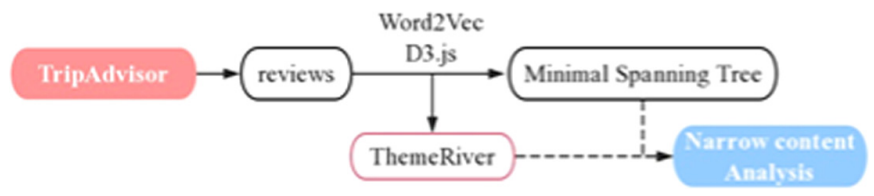

Fig. 1. Data process flow 


\section{MSTs and ThemeRivers for Mu Tianyu Great Wall and Ba Daling Great Wall}

MSTs' different colors are used to distinguish words. The size of the nodes represents word frequency. And the relationship between them is represented by the length of their edges. The closer the relationship, the shorter the edge. The words, lines, and MSFs reveal what tourists' concerns are (Table 1).

Table 1. The MST of Ba daling Great Wall and the MST of Mu tianyu Great Wall

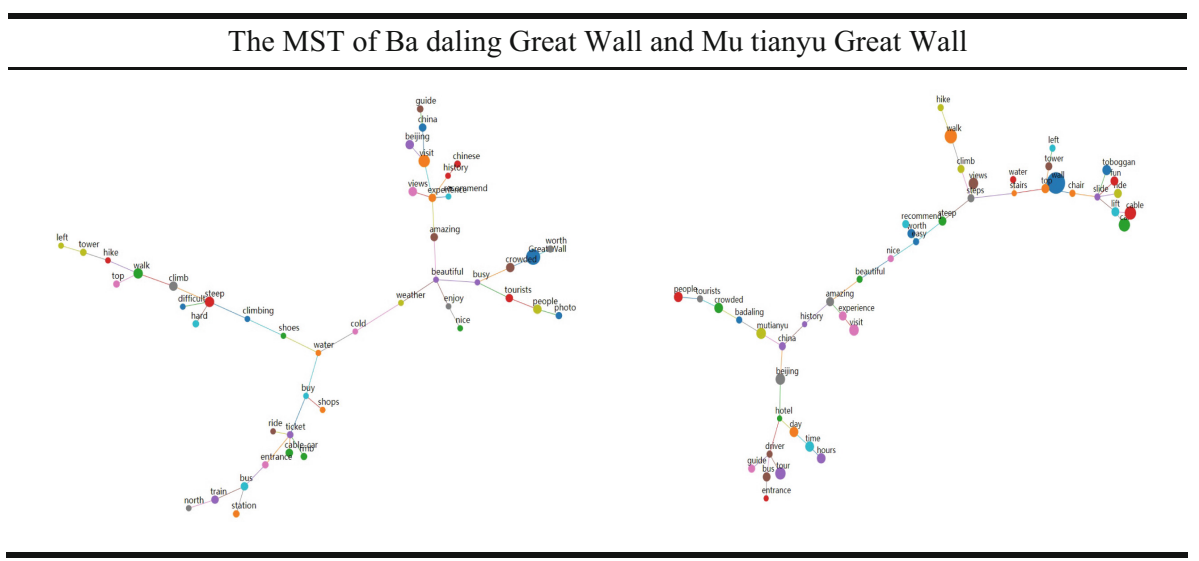

These depictions can only give a roughly ballpark statement of Great Wall. If MST does not apply the information leading to the identification of temporal changes, this research seeks to extend the current knowledge using ThemeRiver. The adjectives of Top 100 of the most common terms are sorted out to act as basic terms for ThemeRiver analysis (Table 2).

The river of Great Walls indicates the following three aspects: (1) the pear-shape flow is formed by the number of reviews in the corresponding month. It clearly shows that April (the spring in Beijing) and September (autumn in Beijing) are the best months to visit the Great Wall. (2) The Great Wall is "hot" in summer and "cold" in winter following Beijing's semi-humid continental monsoon climate in the north temperate zone. (3) "steep" "crowded" and "amazing" are mentioned most in Ba daling Great Wall reviews, and "steep" "crowded" and "worthy" are mentioned most in $\mathrm{Mu}$ tianyu Great Wall reviews. As an outdoor heritage site, the Great Wall is greatly influenced by seasons. 
Table 2. The MST of Ba daling Great Wall and Mu tianyu Great Wall

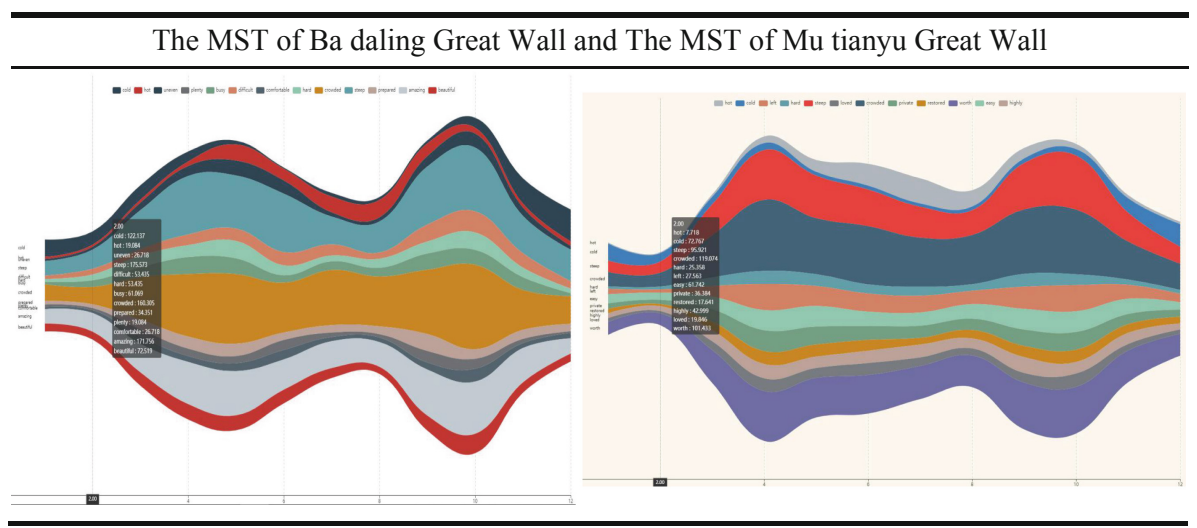

\section{Summary and Managerial Suggestions}

This research seeks to extend the current knowledge by applying MST, ThemeRiver and investigate the differences and similarities of two Great Wall sites from reviews of English-speaking tourists.

In terms of methodology, we applied an innovative research route to combine MST with ThemeRiver to visualize travellers' online comments. At the same time, the visual results obtained are combined with qualitative analysis to generate valuable, intuitive summaries can be used for reference in future research.

Practically, we discovered that both scenic places are "crowded" and "steep", but "amazing" and "worthy", and the best seasons to pay a visit are the spring and autumn. The weather in Great Wall is hot in summer and cold in winter. tourists take experiences of climbing walls as a way of learning Chinese history and culture.

A careful study of the summaries' commonalities can be a good source of insight for management and business promotion. For both heritage sites, Badaling Great Wall and $\mathrm{Mu}$ tianyu Great Wall can be highly praised in marketing promotion. For the crowded situation caused by the tourist season, the managerial department can give the corresponding warning and prompt, and the measures of "flow restriction" to alleviate. In today's Internet era, tourists book tickets in advance by Apps. A recommendation system can be established to remind consumers to prepare mountaineering supplies, prevent heat in summer and cold in winter, and significantly improve tourists' satisfaction through short messages.

On the other hand, careful studies of their differences from summaries can make them learn from and complement each other. Mu tianyu Great Wall is very popular among tourists because of the great convenience provided by the cable car and toboggan. In fact, Ba daling Great Wall is steeper than Mu tianyu Great Wall, if the cable car service can be provided, it is sure to increase tourists' convenience and improve their experience. The guide service of Ba daling Great Wall makes the tourists 
very satisfied. Taking pictures and learning Chinese history are realized with the help of the guide. The managers from Mu tianyu Great Wall can learn from this experience, and actively improve their tour guide team. Besides, tourists are reminded to bring more drinking water when climbing mountains in $\mathrm{Mu}$ tianyu Great Wall. One reason is the huge water consumption during climbing; the other reason is that the price of water is too high in the heritage site, which needs to arouse managers' attention to act.

\section{References}

1. History.com Editors. Great Wall of China. https://www.history.com/topics/ancient-china/ great-wall-of-china (2020)

2. Ashworth G, Page SJ (2010) Urban tourism research: Recent progress and current paradoxes. Tourism Manage 32(1):1-15

3. Kladou S, Mavragani E (2015) Assessing destination image: An online marketing approach and the case of TripAdvisor. J Destination Mark Manage 4(3):187-193

4. Richards G (2018) Cultural tourism: a review of recent research and trends. J Hospitality Tourism Manage 36:12-21

5. Setlur V, Stone MC (2016) A linguistic approach to categorical color assignment for data visualization. IEEE Trans Visual Comput Graph 22(1):698-707

6. Sundberg REA (2012) Visualizing sentiment analysis on a user forum. In: Eighth International European Language Resources Association (ELRA), pp 3573-3579

7. Wang CEA (2013) SentiView: sentiment analysis and visualization for internet popular topics. IEEE Trans Human Mach Syst 43:620-630

8. Barreda A, Bilgihan A (2013) An analysis of user-generated content for hotel experiences. J Hospitality Tourism Technol 4(3)

9. TripAdvisor (2019) Mu tianyu Great Wall. https://www.tripadvisor.co.uk/Attraction Review-g294212-d325811-Reviews-Mutianyu_Great_Wall-Beijing.html 1 November 2019

10. TripAdvisor (2019) The Great Wall at Ba daling. https://www.tripadvisor.co.uk/Attraction Review-g294212-d311505-Reviews-The_Great_Wall_at_Badaling-Beijing.html

11. Blei DM, Lafferty JD (2006) Dynamic topic models. Mach Learn

12. Nicholson C (2019 A beginner's guide to word2vec and neural word embeddings. https:// pathmind.com/wiki/word2vec 12 Nov 2019

Open Access This chapter is licensed under the terms of the Creative Commons Attribution 4.0 International License (http://creativecommons.org/licenses/by/4.0/), which permits use, sharing, adaptation, distribution and reproduction in any medium or format, as long as you give appropriate credit to the original author(s) and the source, provide a link to the Creative Commons license and indicate if changes were made.

The images or other third party material in this chapter are included in the chapter's Creative Commons license, unless indicated otherwise in a credit line to the material. If material is not included in the chapter's Creative Commons license and your intended use is not permitted by statutory regulation or exceeds the permitted use, you will need to obtain permission directly from the copyright holder.

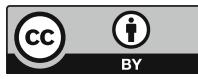

